\title{
A Study on Three-dimensional Effects and Deformation of Textile Fabrics: \\ Dynamic Deformations of Silk Fabrics
}

\author{
Kim Minjin $\cdot$ Kim Jongjun ${ }^{+}$
}

Dept. of Clothing and Textiles, Ewha Womans University

Dept. of Clothing and Textiles, Ewha Womans University ${ }^{+}$

\begin{abstract}
Recent trends toward the collaborations among various sectors of academia and research areas have brought interests and significances in new activities especially in the fashion and textile areas. One of the collaboration examples is the recent research projects on $3 D$ virtual clothing systems based on the 3D CAD software. The $3 D$ virtual clothing systems provide simulated apparels with high degrees of fidelity in terms of color, texture, and structural details. However, since real fabrics exhibit strong nonlinearity, anisotropy, viscoelasticity, and hysteresis, the 30 virtual clothing systems need fine tuning parameters for the simulation process. In this study, characteristics of silk fabrics, which are woven by using degummed silk and raw silk yarns, are being analyzed and compared. Anisotropic properties may be measured as warp and filling direction properties separately in woven fabrics, such as warp tensile stress or filling bending rigidity. Hysteretic properties may be measured as bending hysteresis or shear hysteresis by using KES measurements. These data provide deformation-force relationships of the fabric specimen. Three-dimensional effects obtained when using these characteristic fabrics are also analyzed. The methods to control the three-dimensional appearance of the sewn fabric specimens when utilizing a programmable microprocessor-based motor device, as prepared in this study, are presented. Based on the physical and mechanical properties measured when using the KES equipment, the property parameters are being into a 3-dimensional virtual digital clothing system, in order to generate a virtual clothing product based on the measured silk fabric properties.
\end{abstract}

Key words : doupion silk, dynamic deformation, KES(Kawabata Evaluation System), 30 scanning

Corresponding author: Kim Jongjun, Tel. +82-2-3277-3102, Fax. +82-2-3277-3079

E-mail: jjkim@ewha.ac.kr 
Kim Minjin - Kim Jongjun / A Study on Three-dimensional Effects and Deformation of Textile Fabrics

\section{Introduction}

Collaborations among various academia and research sectors have become very important issues. There have been many scientists, and designers who have shown numerous instances implying the importance of the collaboration between various disciplines. One of the collaboration examples is the recent research projects on $3 \mathrm{D}$ virtual clothing systems based on the $3 D$ CAD software. Physics-based computer simulation of clothing help designers to preview virtual products, with high degrees of fidelity in terms of color, texture, and structural details, prior to preparing it using actual textile fabrics (Ko, Choy, \& Ko, 2009). A recent comparative study on the avatar sizing and actual body measurement shapes reported the differences in detail (Lee \& Sohn, 2012). 3D clothing simulation system was also utilized in the appearance evaluation of men's work clothes jumper pattern making (Park \& Lee, 2012). As the $3 D$ virtual clothing system improved and diversified, the software system may be utilized in a digital fashion show (Wu et al., 2013). With the improvement in the accuracy, the $3 \mathrm{D}$ virtual clothing system may also be employed in calculating the fitting, and amount of air gap in adult women's apparel (Cha \& Kang, 2013). However, since real fabrics exhibit strong nonlinearity, anisotropy, viscoelasticity, the 30 virtual clothing simulation system needs fine tuning parameters for its calculation. The classic approaches include force-based measurements and dynamic captured video analysis (Wang, Hecht, Ramamoorthi, \& O’Brien, 2010).

Together with these innovative approaches, there have also been needs for nurturing the collaboration efforts between these state of the arts sectors and the traditional textile industries.
In the area of 'sustainable growths' in the textile and apparel industries, a group of participants comprise the well-known figure, Safia Minney (Minney, 2012), focusing on ethical trading fashion, founder of People Tree, and Abi and Thomas Petit, founders of Gossypium (Black, 2011).

There have been ceaseless efforts to obtain environment-friendly textile materials. In terms of silk fibers (Nam \& Shin, 1998), the traditional silk variety of 'Oksa' (Doupion silk) is an irregular and thick silk thread reeled from two or more entangled cocoons and producing a coarse yarn with the sericin covering the silk fibroins intact. This type of silk fabric is one of the fabrics which are not subject to the degumming process of removing sericin from the raw silk yarn (Zhu, Arai, \& Hirabayashi, 1995). The degumming process, or scouring needs almost inevitably several chemicals and water. In this process, according to the final needs, the degree of degumming may be appropriately adjusted, thereby controlling the fabric hand, and reducing the amount of chemicals or water.

Degumming, or scouring process needs inevitably several chemicals and water. The fabric hand may be controlled by adjusting the degree of degumming, which may reduce the amount of chemicals or water.

As described above, the textile property parameters for preparing $3 D$ virtual clothing are quite complex. In order to tackle the issues, nonlinearity, anisotropy, viscoelasticity, and hysteresis of the fabrics should be clearly defined and analyzed. As an approach, anisotropic properties may be measured as warp and filling direction properties separately in woven fabrics, such as warp tensile stress or filling bending rigidity. Hysteretic properties may be measured as bending hysteresis or shear 
hysteresis by using KES measurements ("Kato Products", 2012). These data provide deformation-force relationships of the fabric specimens.

In this study, as one of eco-friendly textile materials, silk fabric specimens were selected in order to analyze the physical and mechanical properties. The measured parameters were then fed into the $3 D$ virtual clothing system for preparing garment. Specifically, characteristics of silk fabrics, woven using degummed silk and raw silk yarns, were analyzed and compared. Three-dimensional effects obtainable by using these characteristic fabrics were also analyzed.

The methods to control the three-dimensional appearance of the sewn fabric specimens when utilizing a programmable microprocessor-based motor device, as prepared in this study, are presented. This type of $3 D$ spatial data according to the fabric deformation might further be used in the future $3 D$ virtual clothing system. Based on the physical and mechanical properties measured when using the KES equipment, the property parameters are being fed into a 3-dimensional virtual digital clothing system, in order to generate a virtual clothing product based on the silk fabric properties.

\section{Theoretical Considerations}

Recent trends toward the convergence among various sectors of academia and research subjects have brought interests and significance in the new activities especially in the fashion and textile sectors. One of the activities is the collaboration between the IT and the traditional fashion industries.

As one of the collaboration efforts, E-TAlLOR project was initiated to develop a platform enabling the integration of Virtual-home shopping, advanced Customized Clothing, and European sizing information infrastructures. The 3D virtual garment simulator of the project includes the mechanical model, a collision engine, rendering and animation. The final virtual garment appearance is displayed based on its material properties, including fabric texture, color, and luster (Volino \& Magnenat-Thalmann, 2000a).

One of the issues from the point of view of the fashion and textiles sectors is the realistic and precise modeling of the non-linear viscoelastic behavior of fabrics. Based on the semi-implicit method proposed by Barraf and Witkin (1998), various studies have suggested variations of the method (Kang, Choi, Cho, \& Park, 2000; Volino \& Magnenat-Thalmann, 2000b). Choi and Ko (2002), however, reported on the issues related to the post-buckling instability resulted from the wrinkles. Expression of realistic cloth wrinkle was made possible by the incorporation of their 'immediate buckling assumption.'

However, more often than not, current clothing simulators produce animations of walking models wearing costumes exhibiting rubber-like behaviors, if not highly noticeable. In order to establish improved constitutive models for the cloth deformation, recent study suggested a system which measures complex $3 D$ deformations of fabrics. The system comprises force sensors and actuators together with a stereo computer vision system to analyze the deformation of the fabric specimen in 3D (Miguel, Bradley, Thomaszewski, Bickel, Matusik, Otaduy, \& Marschner, 2012). This system, however, requires 4 cameras and supporting software, 8 linear actuators, and 8 load cells with supplementary equipment to analyze a fabric specimen.

As one area of the IT industries, physical computing (“ITP Physical Computing”, 2012) 
Kim Minjin - Kim Jongjun / A Study on Three-dimensional Effects and Deformation of Textile Fabrics

encompasses interactive physical systems using software and hardware that can sense and respond to the outside world. Physical computing is an interactive framework for understanding people's relationship to the digital world. The term most often describes handmade design or hobby projects using sensors and microcontrollers to translate analog input to the system. It is also possible to control electro-mechanical devices such as motors, servos, lighting or others.

As an open-source microcontroller devices for other disciplines, Arduino (2013) has widely been employed by designers, or various user groups. Arduino is an open-source physical computing platform based on a simple input-output board of small form factor, and a development environment that implements the Processing language. Therefore, Arduino-based interactive system may be incorporated to the textile fabrics or clothing together with a small mechanical device to move the clothing component.

It is difficult to define pliable and easily deformable materials numerically, such as textile fabrics or clothing, in terms of three-dimensional data points. One of the traditional methods include contact point measurement scheme, such as an articulated form contact 3D scanner. The contact $3 \mathrm{D}$ scanners probe the subject through physical touch, while the object is resting on a plate. This type of contact $3 D$ scanner is, therefore, not applicable to the delicate surface of soft textile fabric product. Structured light 3D scanner (Zhang, Weide, \& Oliver, 2010) has gain some popularity in the measurement of soft products.

Objective measurement study on the fabric hand was initiated by Peirce (1930). Kawabata (1980) conducted a comprehensive study of the fabric handle, devising the Kawabata Evaluation System for the objective measurement of handle of textile fabrics in Japan. The methods have been employed in the $3 D$ virtual clothing systems as parameters for fabric physical properties.

In this study, fabric physical properties related to the anisotropy analysis, such as warp and filling stiffness, or B (bending rigidity) of warp and filling directions are separately measured. The hysteresis related parameters, such as $2 \mathrm{HB}$ (bending hysteresis) of warp and filling directions, and $2 \mathrm{HG}$ (shear hysteresis) of warp and filling directions, are also analyzed, in order to help interpret the differences in the real fabric parameters and virtual fabric parameters.

\section{Experimental Methods}

\section{Fabric Specimen Selection}

Four silk fabric specimens, 'Oksa', 'Myungjoo', 'Nobang', and 'Habutai', are selected for experiment. Fabric count, denier, weight per unit area, and thickness are measured for the specimens (Table 1). 'Oksa' and 'Nobang' are woven with raw silk yarns and mostly stiff to the touch, while 'Myungjoo' and Habutai are woven with degummed silk yarns with soft feel to the touch. Photomicrographs of the fabric specimens are shown in Figure 1.

\section{Experimental Methods and Device}

By utilizing the stiff property of silk fabric such as 'Oksa', characteristic three-dimensional effect may be obtained employing a motorized device controlled by a microcontroller. 
Journal of Fashion Business Vol.17, No.6

Table 1. Measurement Results of Fabric Specifications

\begin{tabular}{l|c|c|c|c}
\hline \hline & 'Oksa' & 'Myungjoo' & 'Nobang' & Habutai \\
\hline $\begin{array}{l}\text { Fabric Count } \\
\text { (warp } \times \text { filling) }\end{array}$ & $102 \times 66$ & $93 \times 66$ & $107 \times 102$ & $152 \times 76$ \\
\hline $\begin{array}{l}\text { Denier, den. } \\
\text { (warp } \times \text { filling) }\end{array}$ & $42 \times 154$ & $103 \times 107$ & $29 \times 50$ & $27 \times 167$ \\
\hline Weight/area, g/m & 52.8 & 67.0 & 30.8 & 72.0 \\
\hline Thickness, mm & 0.205 & 0.146 & 0.134 & 0.134 \\
\hline \hline
\end{tabular}

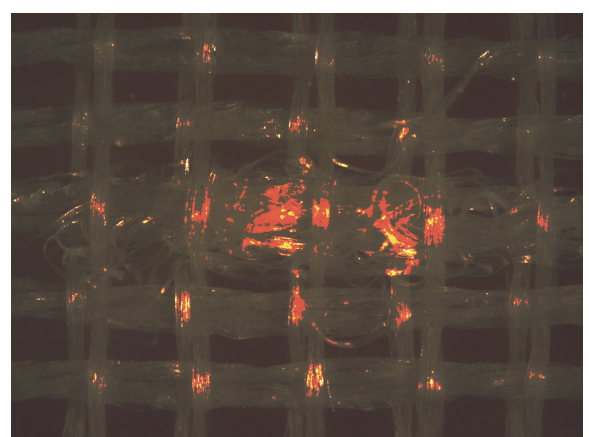

(a) 'Oksa'

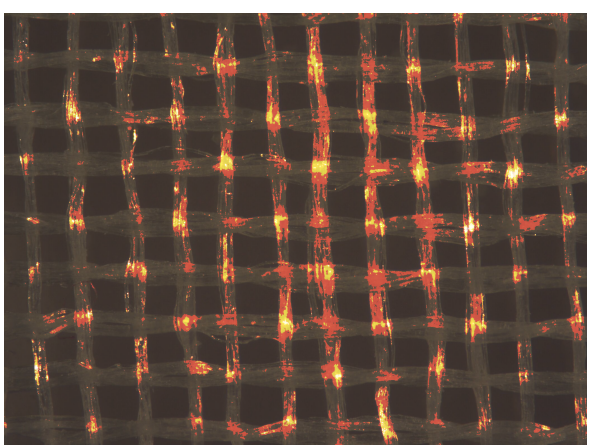

(c) 'Nobang'

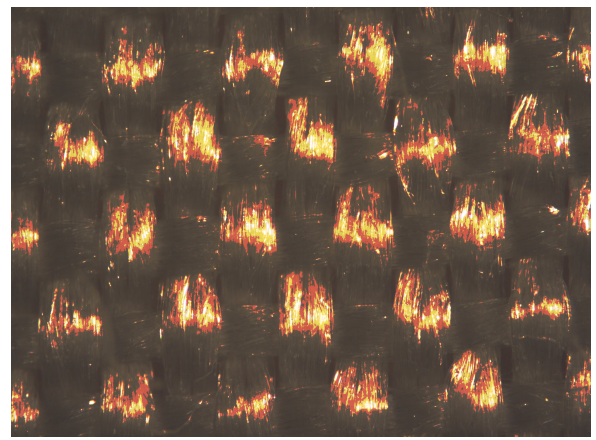

(b) 'Myungjoo'

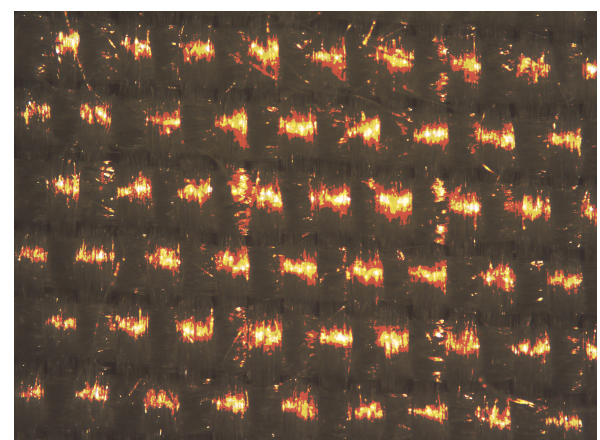

(d) Habutai

Figure 1. Photomicrographs of Fabric Specimens (Objective $=4 \mathrm{X}$ )

Stiffness Measurement using Flexometer Method Flexural rigidity is related to the stiffness feel to the touch. Stiffness of the specimen was measured using Flexometer method. Bending length, or drape stiffness, and flexural rigidity were calculated as follows: 
Bending length $\mathrm{C}=\mathrm{L} / 2(\mathrm{~cm})$

Flexural rigidity $G_{f}=C^{3} \times W(g f \cdot c m)$,

where $W=$ weight per area $\left(\mathrm{gf} / \mathrm{cm}^{2}\right)$.

\section{KES Analysis}

KES instruments were used to measure tensile, bending, surface, and compression properties of fabric specimens. The instruments generate calculated results and graphic output, which is used to characterize the fabric deformation and recovery properties. In order to relate the hand of fabrics to the mechanical and physical measurement, the amount of fabric deformation should be similar to the deformation by the human subjects during the hand evaluation process or the finishing process of the fabrics as much as possible. Fabric specimen size is $20 \times 20 \mathrm{~cm}$.

Tensile test measures the stress and strain parameters at a specified maximum load for the type of material being tested. Tensile measurement block measures EM, extensibility, WT, tensile energy, LT, linearity, and RT, resilience in tensile deformation.

Shear tester applies opposing and parallel forces to the fabric specimen until offset angle of 8 degree is reached. Shearing block measures G, shear stiffness, $2 \mathrm{HG}$, hysteresis at $0.5^{\circ}$, and $2 \mathrm{HG} 5$, hysteresis at $5^{\circ}$.

Bending block measures $\mathrm{B}$, bending rigidity, and $2 \mathrm{HB}$, hysteresis.

Compression properties of a $2 \mathrm{~cm}^{2}$ area of fabric specimen are measured at an appropriate force. Compression block measures LC, linearity, $\mathrm{WC}$, compressional energy, and $\mathrm{RC}$, resilience in compression.

Fabric surface contour and friction properties are determined using surface probe and friction probe made of series of fine steel wires. Surface block measures MIU, coefficient of friction, MMD, mean deviation of MIU, and SMD, geometrical roughness.

\section{Servomotor Device controlled by Arduino}

As a prototype, the specifications of the servo motor and ancillary devices are as follows: maximum rotation angle $400^{\circ}$, rotation speed $1.4 \mathrm{sec} / 60^{\circ}$ (at $4.8 \mathrm{~V}$ ), $7: 1$ reduction gear system as shown in Table 2. One end of the prepared fabric specimen was extended at a constant rate.

In order to control the servo motor, Arduino Uno was employed as a microprocessor. The program code in Table 3 is based on Kurts's (2006) work.

Table 2. Servo Motor Specifications

\begin{tabular}{l|l}
\hline \multicolumn{1}{c|}{ Control and pulse } & \multicolumn{1}{c}{ Detail specifications } \\
\hline Control system and make & $\begin{array}{l}\text { +Pulse Width Control 1500usec Neutral } \\
(\text { Hitec, digital servo, model HS-5485HB })^{\star}\end{array}$ \\
\hline Required pulse & $3-5$ Volt Peak to Peak Square Wave \\
\hline Operating voltage & $4.8-6.0$ Volts \\
\hline \hline
\end{tabular}

*) Hitech HS-5035HD Digital Ultranano model weighs around 4.4g. This needs testing regarding torque and fabric stiffness relationship for this study. 
Table 3. Partial Programming Code for the Arduno - Stepper Motor - Based on Kurts (2006)

\begin{tabular}{|c|c|}
\hline & Arduino code \\
\hline $\begin{array}{l}\text { Program } \\
\text { Heading }\end{array}$ & $\begin{array}{l}\text { * Servo Serial Better } \\
\text { * Created } 18 \text { October } 2006 \\
\text { * copyleft } 2006 \text { Tod E. Kurt <tod@todbot.com> } \\
\text { * http://todbot.com/ }\end{array}$ \\
\hline Main & $\begin{array}{ll}\text { int servoPin }=7 ; & / / \text { Control pin for servo motor } \text {-change as per user's need } \\
\text { int pulseWidth }=0 ; & / / \text { Amount to pulse the servo } \\
\text { long lastPulse }=0 ; & / / \text { the time in millisecs of the last pulse } \\
\text { int refreshTime }=20 ; & / / \text { the time in millisecs needed in between pulses } \\
\text { int val; } & \quad / / \text { variable used to store data from serial port } \\
\text { int minPulse }=700 ; & / / \text { minimum pulse width } \\
& \end{array}$ \\
\hline
\end{tabular}

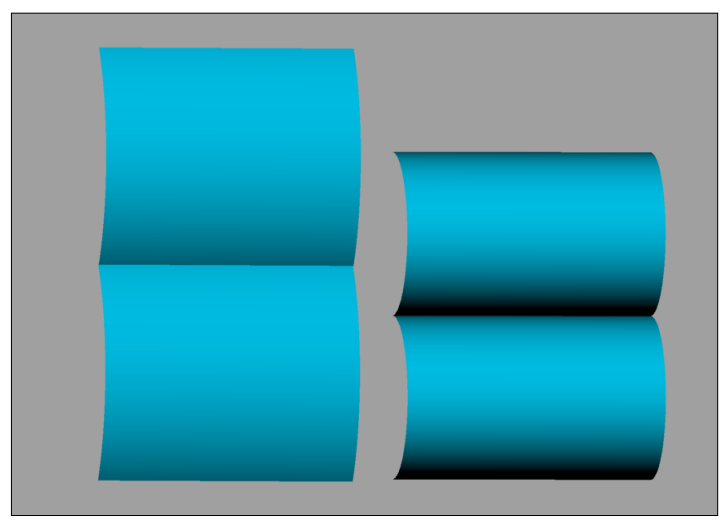

Figure 2. Schematic 3-dimensional Change of a Fabric Specimen,

Left: Before Contraction by Wire, Right: After Contraction

Flexible rope and acrylic pipe for fixation were installed at the sewn section of the fabric specimen. Since the 30 scanning procedure requires a stationary state of the object, the fabric specimen was extended to 1, 2, 3, and $4 \mathrm{~cm}$, respectively, at motionless state as shown in Figure 2.
In case of irregular and extraordinary 30 data, during the scanning procedure, due to the sparse fabric count, excessive surface glare, or fabric color, we selected the special spray (Helling $\mathrm{GmbH}$, Standard-Chek Developer No.3) to improve the data quality obtained using $3 D$ scanning. 

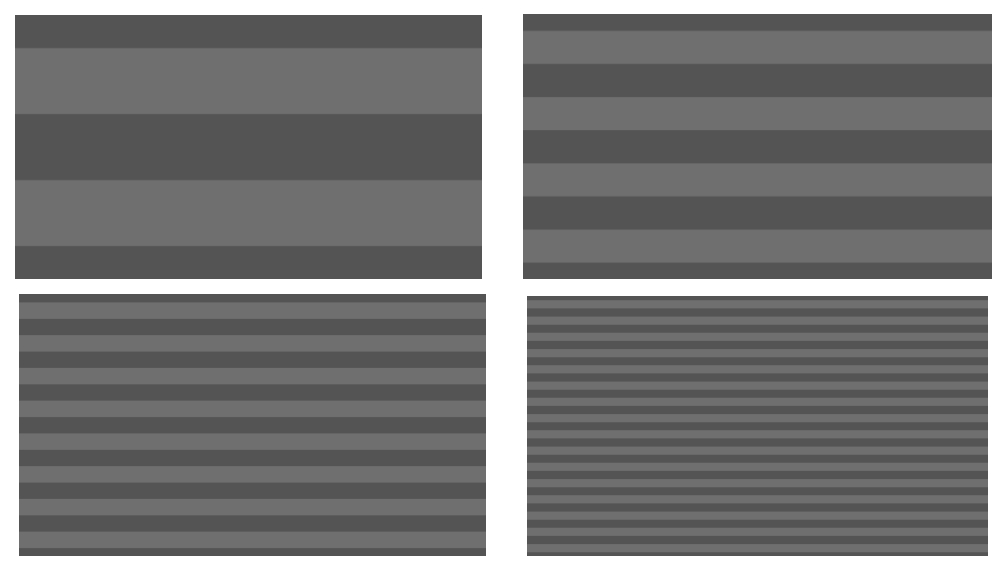

Figure 3. Some Selected Pattern Sequences for 3D Surface Reconstruction

\section{D Surface Scanning System using White Structured Light}

A series of white structured light beam patterns, as shown in Figure 3, were used for three-dimensional scanning and reconstruction. The program used was DAVID-Laserscanner Pro ("DAVID-Laserscanner Pro instruction manual", 2012). Three-dimensional information about the spatially modified fabric specimen was obtained using Rhino3D. Among the program functions, 'PolylineOnMesh' and 'PolylinePoints' functions were employed in order to extrace the values of $(x, y, z)$ axes. The data were then exported to MS Office Excel. For the comparison of the spatial information of the fabric specimen, the data were reconstructed as $2 \mathrm{D}$ axis data.

\section{Results and Discussion}

Silk fabric specimens were analyzed using KES equipment and Flexometer method. Sewn silk fabric specimens were dynamically deformed to achieve three-dimensionally morphed structure due to the specified square patterns and employed sewing method. 3D scanning was implemented to register the point cloud data and surface reconstruction for spatial information of the deformed fabric structure.

\section{KES Shear properties}

During the initial stage of shear deformation of fabric specimen, yarn to yarn friction, relative rotation of the warp and filling yarns at the inter-yarn cross-over point, and bending of yarns contribute to the increase in the shear rigidity of the specimen (Figure 4). At a later stage of the deformation, fabric jamming occurs due to the reduced inter-space between the comprising yarns.

Since 'Oksa' is a loose fabric specimen, the fabric jamming does not seem to occur even at the shear angle of 8 degree, while other specimens such as 'Myungjoo' and Habutai develop jamming at the later deformation stage, judging from the relatively sharp increase of the shear deformation curve around 6 to 8 degree. This sharp increase does not develop in the case of 'Oksa' specimen. 


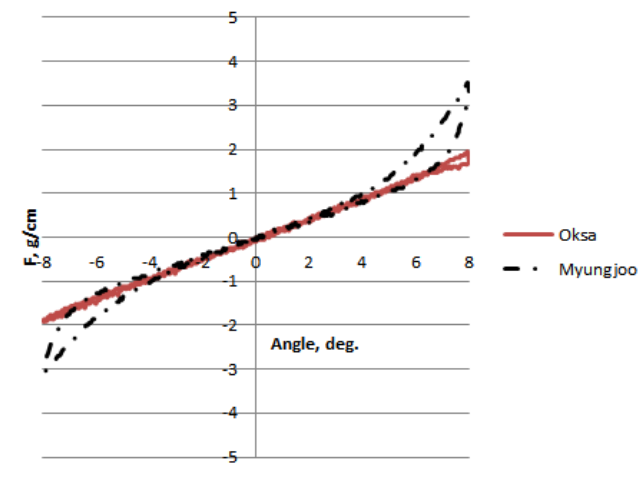

(a) Oksa and Myungjoo

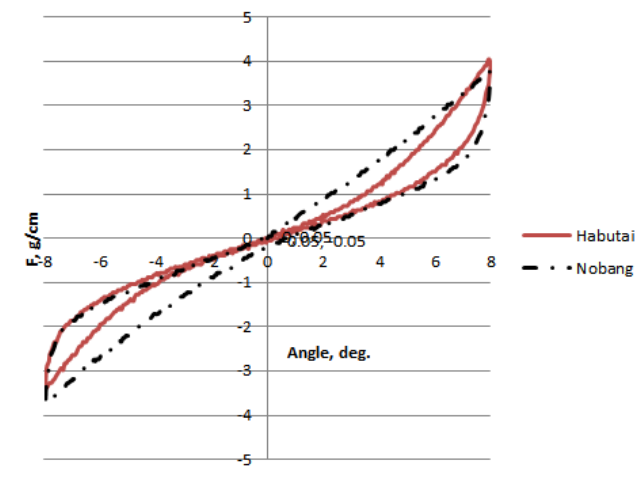

(b) Nobang and Habutai

Figure 4. Shear Chart of Fabric Specimens, Filling Direction

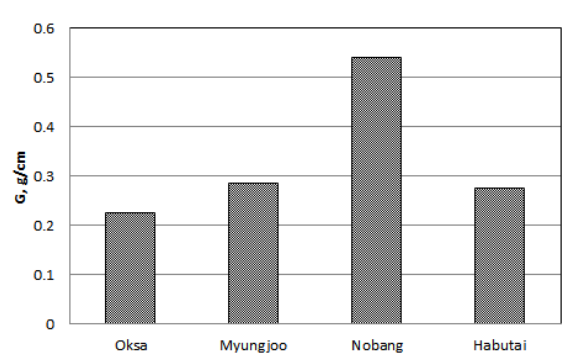

(a) shear rigidity

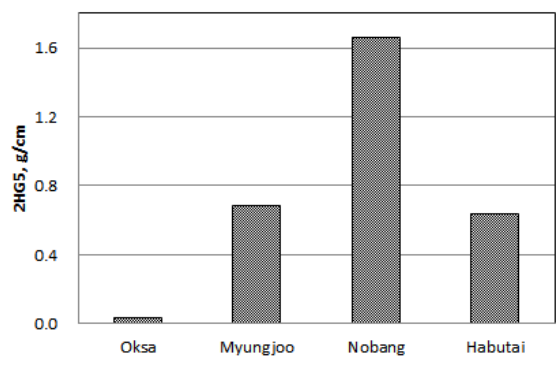

(b) shear hysteresis

Figure 5. (a) Shear Rigidity and (b) Hysteresis at Shear Angle of 5 Degrees

Figure 5 shows the $\mathrm{G}$, shear rigidity, and $2 \mathrm{HG} 5$, shear hysteresis at the degree of 5 . 'Nobang' shows the highest shear rigidity and shear hysteresis, while 'Oksa' shows the lowest shear rigidity and shear hysteresis. It seems that, in the case of 'Nobang', sericin or other components in the raw silk yarns comprising the specimen have increased the shear resistance between the warp and filling yarns. After the first increase in the shear deformation up to 8 degree, the stiff sericin layer between the warp and filling yarns might have been deteriorated. This deterioration might have caused the change in the shear curve leading to the highest shear hysteresis at 5 degree. In the case of 'Oksa', shear rigidity and hysteresis values are the lowest, even though the specimen has the stiffest feel to the touch.

\section{KES Bending properties}

Bending properties are closely related to the fabric stiffness or softness. Higher B value indicates greater stiffness of the fabric specimen. Figure 6 shows the bending curves of 'Oksa' specimen's warp and filling directions. Since the denier difference between the warp (42den.) and filling (154den.) yarns is appreciable, the 
bending rigidity of filling is much higher than that of warp direction. One interesting observation is that the hysteresis value of bending deformation is not high. This seems to be related to the fact that the shear hysteresis of 'Oksa' is also relatively low, suggesting that the level of friction between the crossed yarns may be low, leading to less hysteresis effect between the yarns.
Suh, Yu, Chung, and Kang (2000) reported that the deflection curves of textile materials may be solved based on numerical analysis method as follows:

$$
\mathrm{M}=\Psi(\chi)=\mathrm{k}\left(1-\mathrm{e}^{-\alpha \chi}\right) \quad(\text { eqn. } 1)
$$

Based on the experimental result and the equation, a regression analysis was carried out. Calculation result showed the following values

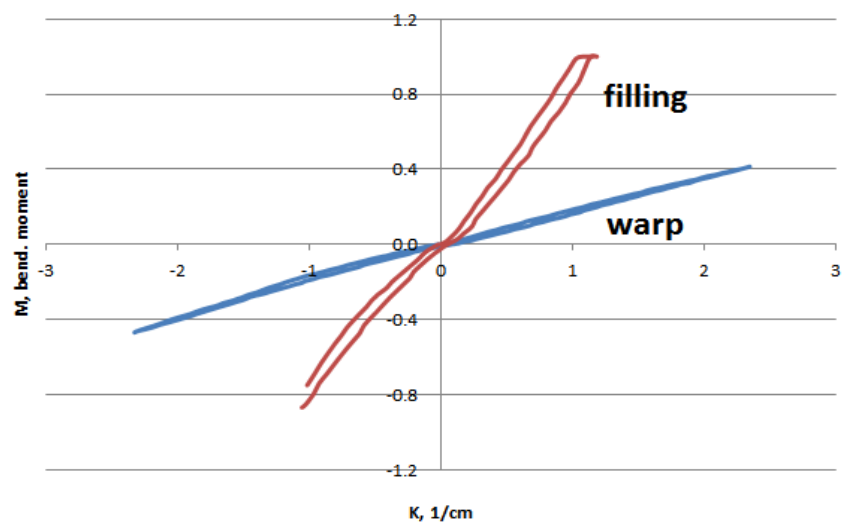

Figure 6. KES Bending Chart of Fabric Specimen 'Oksa', Warp and Filling Direction

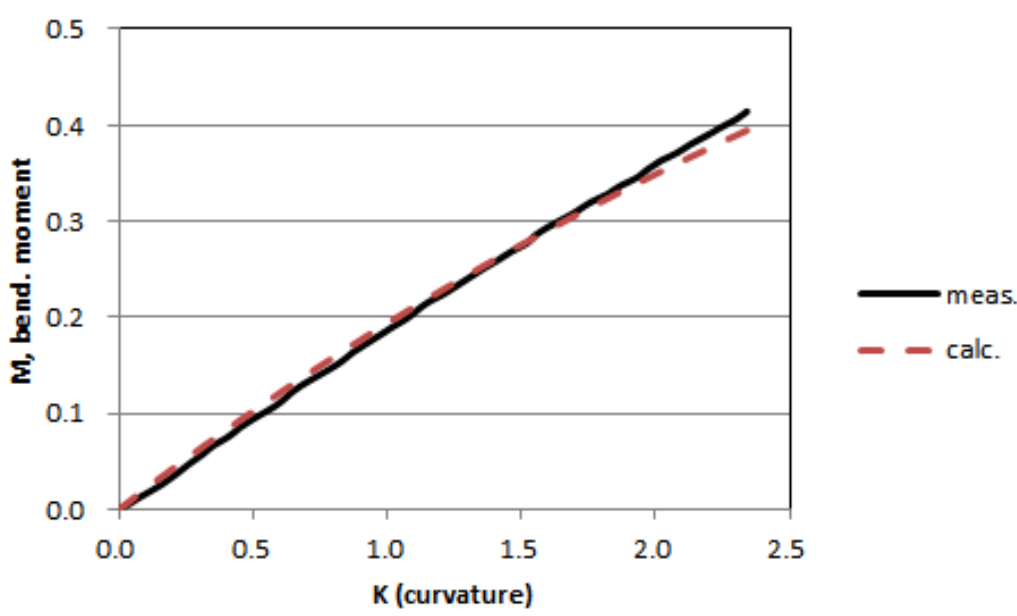

Figure 7. Comparison of Measured and Calculated Values of KES Bending Test, 'Oksa' Warp Direction 


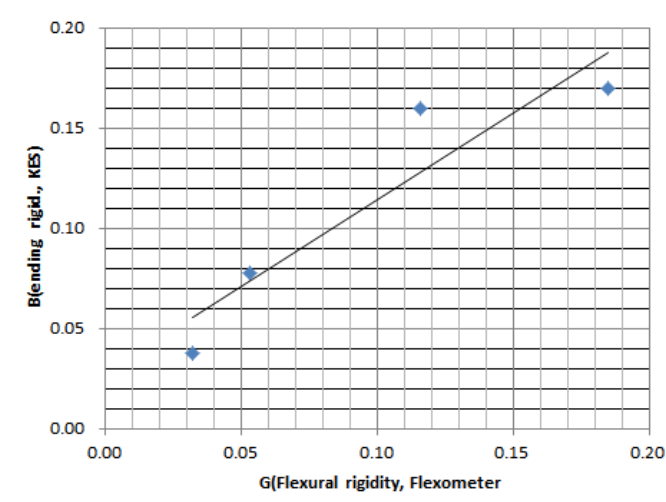

(a) Warp direction

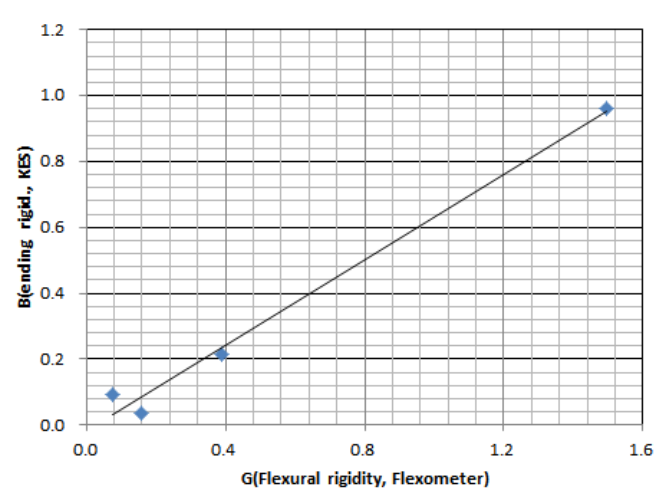

(b) Filling direction

Figure 8. Relation Between the $\mathrm{G}_{f}$ (Flexural Rigidity, Flexometer) and $B$ (Bending Rigidity, KES) : (a) Warp, and (b) Filling Direction

Table 4. Fabric Stiffness Measurement (Bending Length, Flexural Rigidity, and B)

\begin{tabular}{l|c|c|c|c|c|c}
\hline \hline \multirow{2}{*}{ Specimen } & \multicolumn{4}{|c|}{ Flexometer } & \multicolumn{2}{c}{ KES Bending } \\
\cline { 2 - 7 } & $\begin{array}{c}\text { C, warp } \\
(\mathrm{cm})\end{array}$ & $\begin{array}{c}\text { C, filling } \\
(\mathrm{cm})\end{array}$ & $\begin{array}{c}\text { F, warp } \\
(\mathrm{gf} \cdot \mathrm{cm})\end{array}$ & $\begin{array}{c}\text { F, filling } \\
(\mathrm{gf} \cdot \mathrm{cm})\end{array}$ & $\begin{array}{c}\mathrm{B}, \text { warp } \\
(\mathrm{g} \cdot \mathrm{cm} / \mathrm{cm})\end{array}$ & $\begin{array}{c}\mathrm{B}, \text { filling } \\
(\mathrm{gf} \cdot \mathrm{cm} / \mathrm{cm})\end{array}$ \\
\hline 'Oksa' & 0.085 & 0.747 & 0.170 & 1.494 & 0.185 & 0.961 \\
\hline 'Myungjoo' & 0.080 & 0.037 & 0.160 & 0.075 & 0.116 & 0.091 \\
\hline 'Nobang' & 0.039 & 0.194 & 0.078 & 0.388 & 0.053 & 0.214 \\
\hline Habutai & 0.019 & 0.078 & 0.038 & 0.157 & 0.032 & 0.037 \\
\hline \hline
\end{tabular}

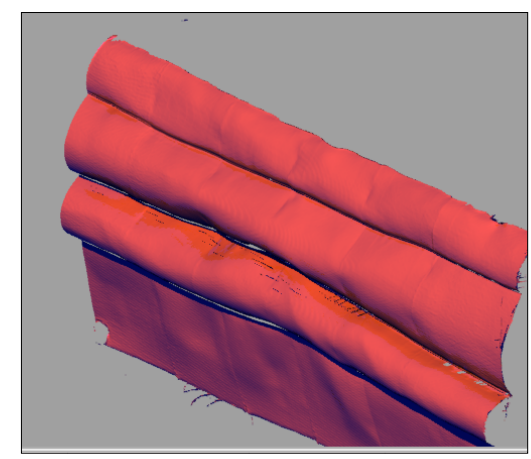

Figure 9. Rendered Image of 3-dimensionally Reconstructed Object Under Contraction Deformation, Specimen: 'Myungjoo' 
for the $k$ and $\alpha$ of in (eqn. 1) with a high correlation coefficient of 0.97 (Figure 7 ).

$\mathrm{M}=\Psi(\chi)=0.98\left(1-\mathrm{e}^{-0.22 x}\right), \quad \mathrm{R}=0.97$

3. Relation between Flexometer Method and KES Bending Analysis
Correlation analysis results between the flexural rigidity by the Flexometer method and the bending rigidity by the KES bending are listed in Table 4. Figure 8 shows that the coefficients of correlation are 0.93 for warp, and 0.99 for filling direction.

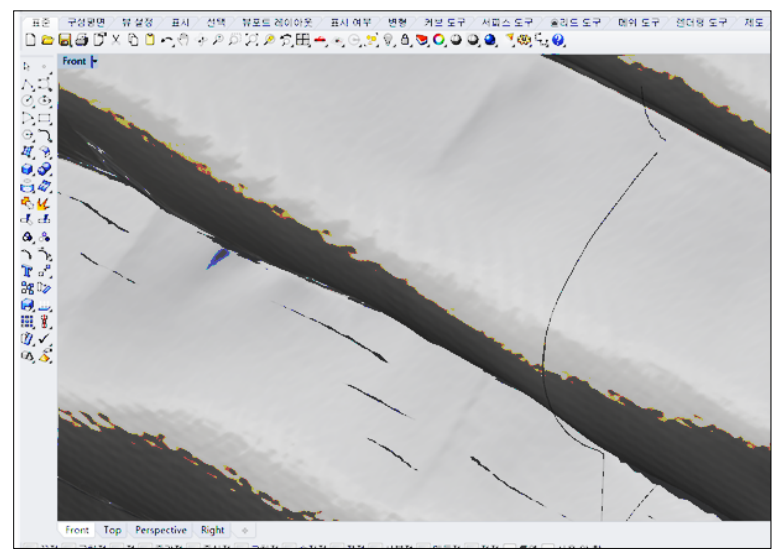

Figure 10. Analysis of Surface Contour of 3-dimensionally Reconstructed Object Under Contraction Deformation, Specimen: 'Myungjoo'

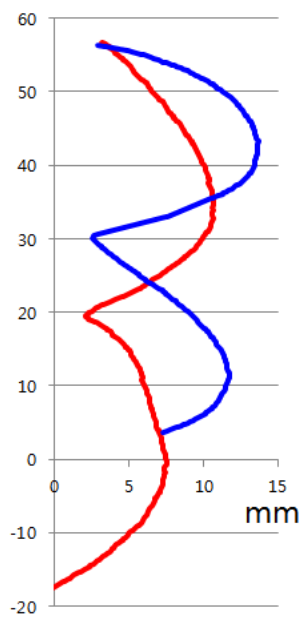

Figure 11. Surface Contour Lines of Contracted Fabric Specimen, 'Oksa' (0mm: Red Line, 40mm: Blue Line) 


\section{Analysis based on 3D Surface Reconstruction}

Figure 9 shows rendered image of 3-dimensionally reconstructed fabric specimen under contraction deformation. Figure 10 shows the surface contour lines on 3-dimensionally reconstructed object using Rhino3D CAD software. The contour line data was exported to MS Excel spreadsheet for further analysis.

Figure 11 shows the contour lines of the sewn fabric specimens contracted by applying the servo motor apparatus, with higher contour line height compared to the one before contraction. This is due to the specimen stiffness, which enables the height increase without too much downward deflection by gravity. The 3D point data may further help compare the discrepancy between the actual fabric deformation and simulated data points from the $3 D$ virtual clothing system.

\section{3D Virtual Digital Clothing Example}

Data for the texture and physical property parameters, such as specular reflection color, glare, opacity, bending, internal damping, etc., were properly typed into the calculation table as shown in Figure 12, Figure 13 shows the application example of simulated 'Oksa' texture on the prepared square fabric patches using a 30 digital clothing software system.

The simulated virtual digital clothing also shows semi-transparent structure similar to the 'Oksa' silk fabric visual effects. The effect reflects 'Oksa'-specific semi-transparency showing the avatar model's contour lines, and the $3 \mathrm{D}$ effects around seam lines, which alter the flat surface to interesting spatial effects. These effects would not be exhibited in the case of 'Myungjoo', which is pliable and opaque.

\section{Conclusion}

Based on the measured parameters of physical and mechanical properties of silk fabrics, deformation behavior of the fabrics, including bending, shear and tensile properties, were analyzed. Anisotropic property were measured as warp and filling direction properties separately in woven fabric, including warp tensile stress or filling bending rigidity. Hysteretic properties were measured as bending hysteresis or shear hysteresis using KES measurement. These data provide deformation-force relationship of the fabric specimen. Comparisons of the degummed silk fabrics and raw silk fabrics were performed. The differences in the physical properties and mechanical properties resulted in the three-dimensional deformation changes.

The methods to control the three-dimensional appearance of the sewn fabric specimens utilizing a programmable microprocessor-based motor device, prepared in this study, were presented. Based on the physical and mechanical properties measured using the KES equipment, property parameters were fed into a 3-dimensional virtual digital clothing system, and generated a virtual clothing product based on the measured silk fabric properties. The relationship between the Flexometer method and KES method has been confirmed.

The results from this study need further refinement in terms of measurement methods and analysis methods, together with the incorporating methods into the $3 D$ virtual clothing system. One of the schemes to improve the cloth simulation models may be the measurement 
Kim Minjin - Kim Jongjun / A Study on Three-dimensional Effects and Deformation of Textile Fabrics

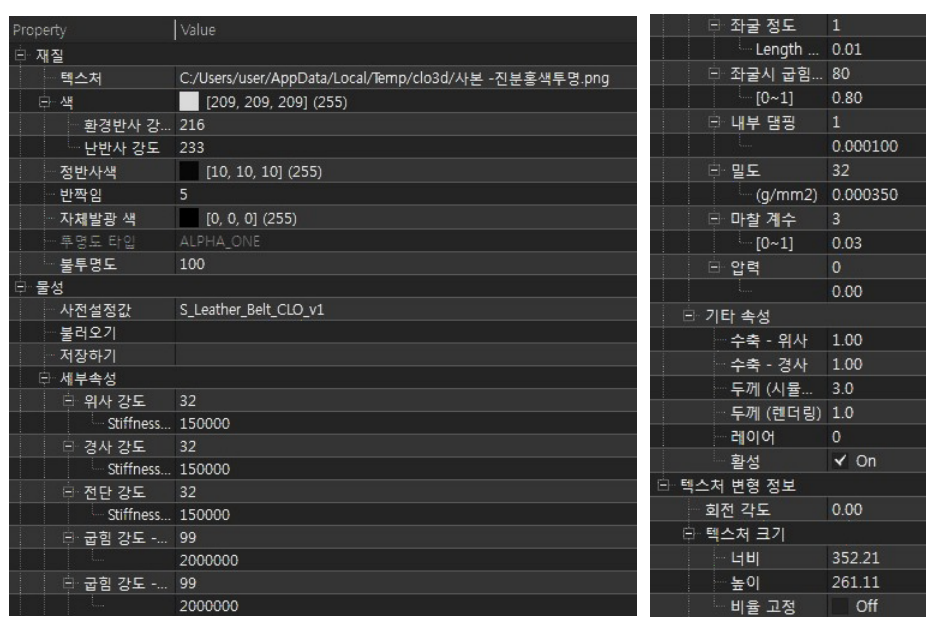

Figure 12. Application of Texture and Physical Property Parameters
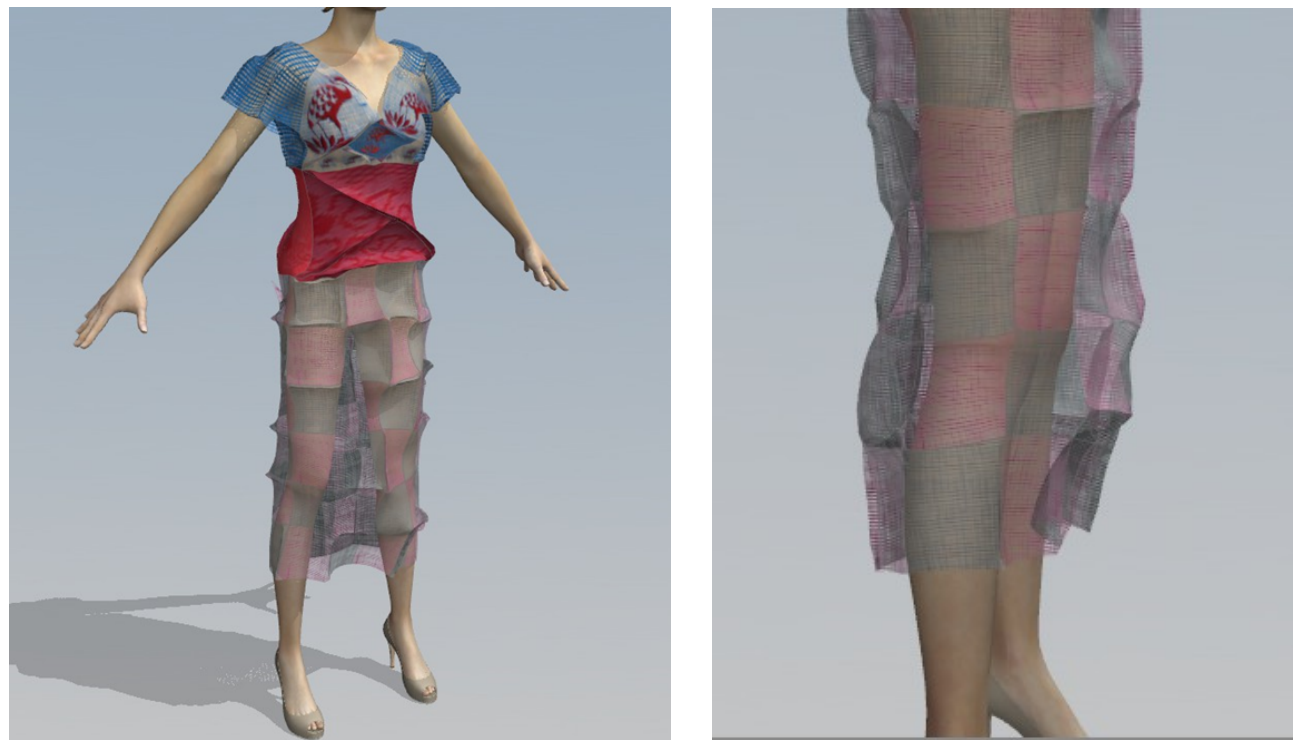

Figure 13. Application Example of 'Oksa' Texture on the Prepared Patches

and analysis of complex 3D deformations of fabric specimens including anisotropy, viscoelasticity, and other time-dependent properties.

Along with the three-dimensional effects of the silk fabrics, the changes accompanying dynamic deformation may be utilized to impart the garment characteristic effect. The current prototype servo motor device used in this study is too bulky to fit into an actual clothing. This issue may be alleviated to some degree by using much smaller and lighter micro servomotor in the next phase of the study. This measure, however, may still pose some problems in terms 
of wearer fitness and comfort. Therefore, collaborations among experts in the specific fields are necessary in the future. Also, the specific behavior of torque or force involved in the movement should carefully be analyzed in the subsequent studies for the optimum size selection.

\section{References}

Arduino. (2012). Getting started with Arduino. Retrieved January 9, 2012, from http://arduino.cc/en/Guide/HomePage

Baraff, D., \& Witkin, A. (1998). Large steps in cloth simulation. Proceedings of SIGGRAPH 1998 (pp. 43-54). New York: ACM New York.

Black, S. (2011). Eco-chic, the fashion paradox. London, UK; Black Dog Publishing.

Cha, S., \& Kang, Y. (2013). Comparison of basic bodice block for adults women by 30 simulation. Journal of Fashion Business, 17(2), 63-81.

Choi, K., \& Ko, H. (2002). Stable but responsive cloth. Proceedings of ACM SIGGRAPH (pp. 604-611). New York: ACM New York.

DAVID-Laserscanner Pro instruction manual. (2012). David Vision Systems. Retrieved October 9, 2012, from http://www.david-3d.com

ITP Physical Computing. (2012). Tisch ITP. Retrieved November 8, 2012, from http://itp.nyu.edu/physcomp/Tutuorials

Kang, Y., Choi, J., Cho, H., \& Park, C. (2000). Fast and stable animation of cloth with an approximated implicit method. Computer Graphics International 2000, 247-256.

Kato Tech Products. (2012). Kato Tech Co. Ltd. Retrieved November 23, 2012, from http://english.keskato.co.jp/products/

Kawabata, S. (1980). The standardization and analysis of hand evaluation. (2nd ed.). Osaka, Japan: The Textile Machinery Society of Japan.

Ko, Y., Choy, H., \& Ko, H. (2009). Evaluation of the clothing simulation technology in the aspects of color, material, structural details, and silhouettes. ITAA Proceedings (pp. 661-664). Washington: International Textile and Apparel Association.

Kurts, T. (2006). Servo serial better. Retrieved March 21, 2013, from http://todbot.com/ arduino/sketches/ServoSerialBetter/ServoSerialB etter.pde

Lee, M., \& Sohn, H. (2012). A comparative analysis of the difference between CLO 3D avatar sizing and actual body measurement shapes. Journal of Fashion Business, 16(4), 137-151.

Miguel, E., Bradley, D., Thomaszewski, B., Bickel, B. , Matusik, W., Otaduy, M. A., \& Marschner, S. (2012). Data-driven estimation of cloth simulation models. Eurographics 2012, 31(2), 1-10.

Minney, S. (2012). People tree. Retrieved December 18, 2012, from http://www.peopletree.co.uk

Nam, J., \& Shin, B. (1998). Silk science. Seoul, Korea: Seoul National University Publishing.

Park, G., \& Lee, W. (2012). Men's work clothes jumper pattern-making and its appearance evaluation through $3-D$ clothing simulation. Journal of Fashion Business, 16(1), 103-120.

Peirce, F. (1930). The 'handle' of cloth as a measurable quality. Journal of the Textile Institute, 21(9), 377-416.

Suh, J., Yu, W., Chung, K., \& Kang, T. (2000). Deflections of fabrics based on nonlinear bending properties. Journal of Korean Fiber Society, 37(5), 286-292.

Volino, P., \& Magnenat-Thalmann, N. (2000a). Virtual clothing-theory and practice. 
Heidelberg. Germany: Springer Berlin.

Volino, P., \& Magnenat-Thalmann, N. (2000b). Implementing fast cloth simulation with collision response. Proceedings of the Conference on Computer Graphics International (pp. 257-266). Geneva: Computer Graphics International.

Wang, H., Hecht, F., Ramamoorthi, R., \& O'Brien, J. (2010). Example-based wrinkle synthesis for clothing animation. Proceedings of ACM SIGGRAPH (pp. 1-8). New York: ACM New York.
Wu, S., Kang, Y., Ko, Y., Kim, A., Kim N., \& Ko, H. (2013). A study on the case analysis and the production of $3 D$ digital fashion show. Journal of Fashion Business, 17(1), 64-80.

Zhang, S., Weide, D., \& Oliver, J. (2010). Superfast phase-shifting method for 3-D shape measurement. Optics Express, 18(9), 9684-9689.

Zhu, L., Arai, M., \& Hirabayashi, K. (1995). Relationship between adhesive properties and structure of sericin in cocoon filament. Journal of Sericulture Science Japan, 64(5), 420-426.

Received(Oct. 22, 2013)

Revised(Nov. 25, 2013; Dec. 10, 2013)

Accepted(Dec. 13, 2013) 